Archived version from NCDOCKS Institutional Repository http://libres.uncg.edu/ir/asu/

\title{
Appalachỉan
}

B O O N E, N O R T H C A R O L I N A

\section{Inflammation And Oxidative Stress Are Lower In Physically fit And Active Adults}

\author{
Authors: \\ R. A. Shanely, D. C. Nieman, D. A. Henson, F.Jin, A. M. Knab, W.Sha
}

\begin{abstract}
The objective of this study was to determine if the inverse relationship between perceived physical fitness (pFIT) and exercise frequency (ExFreq) levels and chronic inflamma-tion and oxidative stress exists after making statistical adjustments for confounders including body mass index (BMI), age, gender, and cigarette smoking. Study partici-pants $(60 \%$ female and $40 \%$ male; $\mathrm{n}=$ 998) varied widely in age (18-85 years) and BMI (16.7-52.7 kg/m2) completed an extensive medical/health and lifestyle questionnaire, and data were used to establish pFIT and ExFreq tertiles. Biomarkers included serum C-reactive protein (CRP), total blood leukocytes, five plasma cytokines [interleukin (IL)-6, IL-10, tumor necrosis factor-a (TNF-a), monocyte chemoattractant protein-1 (MCP1), and granulocyte colony-stimulating factor (GCSF)], F2-isoprostanes, ferric reducing ability of plasma (FRAP), and oxygen radical absorbance capacity (ORAC). A general linear model was used to examine relationships between pFIT and ExFreq with inflammation and oxidative stress while controlling for age, gender, BMI, and smoking. Benjamini-Hochberg method for false discovery rate correction was used for multiple testing corrections. Significant tests $(\mathrm{P}<0.05)$ for trend were found for the effect of pFIT and ExFreq on CRP, white blood cell,

IL-6, TNF-a, GCSF, and F2-isoprostanes, but not MCP1, IL-10, FRAP, and ORAC, after

adjustment for confounders. These data indicate that an inverse relationship exists among chronic inflam-mation, oxidative stress, and pFIT and ExFreq at the community level even after adjustment for important confounders.
\end{abstract}

R. A. Shanely, D. C. Nieman, D. A. Henson, F.Jin, A. M. Knab, W.Sha (2011) "Inflammation and oxidative stress are lower in physically fit and active adults." Scandanavian Journal of Medicine \& Science In Sports Version of Record Available @ www.onlinelibrary.wiley.com (doi: 10.1111/j.1600-0838.2011.01373.x) 


\title{
Inflammation and oxidative stress are lower in physically fit and active adults
}

\author{
R. A. Shanely ${ }^{1,2}$, D. C. Nieman ${ }^{1,2}$, D. A. Henson ${ }^{3}$, F. Jin ${ }^{1,2}$, A. M. Knab ${ }^{1,2}$, W. Sha ${ }^{4}$ \\ ${ }^{1}$ Human Performance Laboratory, Appalachian State University, North Carolina Research Campus, Kannapolis, North Carolina, USA, \\ ${ }^{2}$ College of Health Sciences, Appalachian State University, Boone, North Carolina, USA, ${ }^{3}$ Department of Biology, Appalachian State \\ University, Boone, North Carolina, USA, ${ }^{4}$ Bioinformatics Service Division, University of North Carolina at Charlotte, North Carolina \\ Research Campus, Kannapolis, North Carolina, USA \\ Corresponding author: R. Andrew Shanely, PhD, Human Performance Laboratory, North Carolina Research Campus, Plants for \\ Human Health Institute, 600 Laureate Way, Kannapolis, North Carolina 28081, USA. Tel: 704.250.5357, Fax: 704.250.5428, E-mail: \\ shanelyra@appstate.edu
}

The objective of this study was to determine if the inverse relationship between perceived physical fitness ( $\mathrm{pFIT})$ and exercise frequency (ExFreq) levels and chronic inflammation and oxidative stress exists after making statistical adjustments for confounders including body mass index (BMI), age, gender, and cigarette smoking. Study participants $(60 \%$ female and $40 \%$ male; $n=998)$ varied widely in age (18-85 years) and BMI $\left(16.7-52.7 \mathrm{~kg} / \mathrm{m}^{2}\right)$ completed an extensive medical/health and lifestyle questionnaire, and data were used to establish pFIT and ExFreq tertiles. Biomarkers included serum C-reactive protein (CRP), total blood leukocytes, five plasma cytokines [interleukin (IL)-6, IL-10, tumor necrosis factor- $a$ (TNF- $a$ ), monocyte chemoattractant protein-1 (MCP1), and granulocyte colony-stimulating factor $(\mathrm{GCSF})], \boldsymbol{F}_{2}$-isoprostanes, ferric reducing ability of plasma (FRAP), and oxygen radical absorbance capacity (ORAC). A general linear model was used to examine relationships between pFIT and ExFreq with inflammation and oxidative stress while controlling for age, gender, BMI, and smoking. Benjamini-Hochberg method for false discovery rate correction was used for multiple testing corrections. Significant tests $(P<0.05)$ for trend were found for the effect of pFIT and ExFreq on CRP, white blood cell, IL-6, TNF- $a$, GCSF, and $F_{2^{-}}$ isoprostanes, but not MCP1, IL-10, FRAP, and ORAC, after adjustment for confounders. These data indicate that an inverse relationship exists among chronic inflammation, oxidative stress, and pFIT and ExFreq at the community level even after adjustment for important confounders.
Physical inactivity has been linked to numerous chronic diseases or chronic disease risk factors (Booth et al., 2000). Atherosclerosis, type 2 diabetes, metabolic syndrome, chronic obstructive pulmonary disease, arthritis, and osteoporosis are associated with chronic low-grade inflammation and oxidative stress (Morrow, 2005; Basu, 2008; Beavers et al., 2010). Physical activity has potential benefits on systemic inflammation and oxidative stress biomarkers frequently employed to study the likelihood of developing these disease states. The acute phase protein $\mathrm{C}$-reactive protein (CRP) is the most frequently measured inflammation biomarker in physical activity-related studies, with others also analyzing total blood leukocytes [white blood cell (WBC)], cytokines interleukin (IL)-6, and tumor necrosis factor- $a$ (TNF- $a$; Beavers et al., 2010). Few data are available on the relationship between physical activity and other inflammation-related cytokines such as granulocyte colony-stimulating factor (GCSF), monocyte chemoattractant protein-1 (MCP1), and the anti-inflammatory cytokine IL-10 (Suzuki et al., 2002). The linkage between physical activity and $F_{2}$-isoprostanes, a widely used and highly regarded biomarker of oxidative stress, is also not well established (Morrow, 2005; Vincent \& Taylor, 2006; Fisher-Wellman et al., 2009).

Systemic inflammation and oxidative stress are at lower levels in groups with higher fruit and vegetable intake and lower body mass index (BMI; Esmaillzadeh et al., 2006; Vincent \& Taylor, 2006; Selvin et al., 2007; Holt et al., 2009). The association of physical activity to inflammation and oxidative stress is equivocal (Vincent \& Taylor, 2006; Fisher-Wellman et al., 2009; Beavers et al., 2010). Most randomized-controlled exerciseintervention studies have yet to demonstrate that inflammation and oxidative stress are decreased in the absence of weight loss despite increases in exercise capacity (Kelley \& Kelley, 2006; Stewart et al., 2007; 2010; Arsenault et al., 2009; Beavers et al., 2010; Church et al., 2010; Thompson et al., 2010). Conversely, other well-controlled exercise training studies have 
reported decreases in inflammation in both diseased (Kadoglou et al., 2007) and healthy (Kohut et al., 2006) subjects independent of weight change. Cross-sectional studies consistently show inverse relationships between biomarkers of systemic inflammation and physical activity or fitness (Abramson \& Vaccarino, 2002; Ford, 2002; King et al., 2003; Colbert et al., 2004; Elosua et al., 2005; Pitsavos et al., 2005), with little information available regarding oxidative stress.

Available cross-sectional studies have not measured the relationship of perceived physical fitness (pFIT) and/or leisure-time exercise frequency (ExFreq) levels on both inflammation and oxidative stress using a variety of outcome measures in groups ranging widely in age and BMI. The purpose of this study was to determine if inflammation and oxidative stress are inversely related to physical activity and fitness in a heterogeneous group of subjects after adjustment for potential confounders.

\section{Methods}

\section{Subjects}

Study participants $(n=998 ; 60.4 \%$ female and $39.6 \%$ male; $18-85$ years of age; BMI of $16.7-52.7 \mathrm{~kg} / \mathrm{m}^{2}$ ) were recruited via mass advertising from the community. Subjects had to be noninstitutionalized, and women were excluded if pregnant or lactating. No other exclusion criteria were employed, and both diseased and non-diseased subjects were admitted into the study, with monitoring of disease status and medication use. Reports have been published from this study, with a focus on quercetin supplementation, inflammation, and oxidative stress (Heinz et al., 2010; Shanely et al., 2010). Quercetin supplementation at two doses $(500 \mathrm{mg} /$ day and $1000 \mathrm{mg} / \mathrm{day}$ ) had no influence on immune function, upper respiratory tract infection, oxidative stress or plasma antioxidant capacity (Heinz et al., 2010; Shanely et al., 2010), and the data from the same subjects were reanalyzed to investigate the influence of pFIT and ExFreq on biomarkers of inflammation, oxidative stress, and plasma antioxidant capacity after adjustment for important confounders. There was no statistical difference in the distribution of supplement subjects among the pFIT or ExFreq tertiles $(P=0.44$, chi-square test). Written informed consent was obtained from each subject, and the Appalachian State University institutional review board approved all experimental procedures.

\section{Study design}

Two weeks prior to the start of the study, subjects provided demographic and lifestyle habit information using a survey posted on the website http://surveymonkey.com (Portland, Oregon, USA). Subjects without Internet access were mailed with a printed version of the questionnaire, with instructions to complete all questions prior to their first lab session. Subjects reported to the lab at the beginning and end of the 12-week period, with height and body mass measured with a stadiometer and an electric scale (Tanita, Arlington Heights, Illinois, USA), respectively. Pre- and post-study height and body mass data were averaged, with BMI calculated as $\mathrm{kg} / \mathrm{m}^{2}$.

pFIT levels were reported in response to the question, "In general, compared to other persons your age, rate how physically fit you are." Subjects responded using a 10-point Likert scale, with 1 denoting "not at all physically fit," 5 denoting "somewhat physically fit," and 10 denoting "extremely physically fit," similar to Gerber et al. (2010). Responses by subjects to this question correlate well with measures of objective physical fitness, perceived well-being, and sleep habits (Plante et al., 1998). Subjects were grouped into pFIT tertiles, with $1-5$ corresponding to low fitness, 6-7 corresponding to medium fitness, and 8-10 corresponding to high fitness.

Leisure-time ExFreq habits were assessed through answers to this categorical question taken from the National Health Interview Survey [National Center for Health Statistics (U.S.), 1999]: "Outside of your normal work or daily responsibilities, how often do you engage in exercise that at least moderately increases your breathing and heart rate, and makes you sweat, for at least $20 \mathrm{~min}$ [such as brisk walking, cycling, swimming, jogging, aerobic dance, stair climbing, rowing, basketball, racquetball, vigorous yard work (gardening), etc.]." Response categories included seldom or never, less than $1,1-2,3-4$, or 5 or more times per week. Subjects were grouped into leisure-time aerobic ExFreq tertiles of $<1,1-4$, or 2::5 times per week. Milton et al. (2011) demonstrate that single-item physical activity questions objectively measure activity levels when compared with other instruments.

\section{Outcome measures}

Each subject provided two overnight-fasted blood samples (between 7 a.m. and 9 a.m.) separated by 12 weeks; plasma biomarkers were averaged from the two blood samples. Blood samples were centrifuged in sodium heparin or ethylenediaminetetraacetic acid tubes, and plasma was aliquoted, immediately flash-frozen in liquid nitrogen, and then stored at $-80^{\circ} \mathrm{C}$ until analysis.

Serum CRP (high sensitivity) was measured in a clinical laboratory using an LX-20 clinical analyzer (Beckman, Brea, California, USA) (Nieman et al., 2007) and has an intra-assay coefficent of variation $(\mathrm{CV})$ of $<5 \%$. Enzyme-linked immunosorbent assays were used to measure total plasma concentrations of IL-6 (high sensitivity), GCSF (high sensitivity), MCP1, TNF- $a$, and IL-10 (high sensitivity) in accordance with the manufacturer's protocols (R\&D Systems, Inc., Minneapolis, Minnesota, USA). Each subject sample and the standards provided by the manufacturer were analyzed in duplicate (Nieman et al., 2005; Nieman et al., 2007). Within-subject samples were analyzed on the same assay plate to decrease inter-kit assay variability, and the intra-assay CV for all variables was less than $10 \%$. The minimum detectable concentration of IL-6 (high sensitivity) was $<0.039 \mathrm{pg} / \mathrm{mL}$, GCSF (high sensitivity) $<0.8 \mathrm{pg} / \mathrm{mL}$, MCP1 $<5.0 \mathrm{pg} / \mathrm{mL}$, TNF$a<0.106 \mathrm{pg} / \mathrm{mL}$, and IL-10 (high sensitivity) $<0.2 \mathrm{pg} / \mathrm{mL}$. Data were analyzed with SOFTmax software (Molecular Devices, Sunnyvale, California, USA). A complete blood count with leukocyte differential was analyzed in the Watauga Medical Center Clinical Hematology Laboratory (Boone, North California, USA), and the WBC count has a $\mathrm{CV}$ of $<2 \%$.

Plasma $F_{2}$-isoprostanes, a measure of oxidative stress, were determined as previously described (Shanely et al., 2010). Plasma samples were used to extract free $F_{2}$-isoprostanes with deuterated $\left[{ }^{2} \mathrm{H}_{4}\right]$ prostaglandin $F_{2 a}$ added as an internal standard. The mixture was then added to a C18 Sep Pak column (Waters Corporation, Milford, Massachusetts, USA), followed by silica solid-phase extractions. $F_{2}$-isoprostanes were converted to pentafluorobenzyl esters, subjected to thin-layer chromatography, and converted to trimethylsilyl ether derivatives. Samples were analyzed by a negative ion chemical ionization gas chromatography-mass spectroscopy (GC-MS) using an Agilent 6890N gas chromatography interfaced to an Agilent 5975B inert MSD mass spectrometer (Agilent Technologies, Inc., Santa Clara, California, USA). The $F_{2}$-isoprostanes assay has detection limit of $\sim 5 \mathrm{pg}$, and the $\mathrm{CV}$ is $<10 \%$. Plasma antioxidant capacity was determined by two independent measures; the ferric reducing ability of plasma (FRAP) assay and the oxygen radical absorbance capacity (ORAC). The detection limits and CVs of the FRAP and ORAC assays are 100$1000 \mathrm{mmol} / \mathrm{L}$ and $6.25-100 \mathrm{mmol} / \mathrm{L}$ and $<10 \%$ and $<5 \%$, respectively (Shanely et al., 2010). 
Table 1. Tertiles of subject perceived physical fitness and leisure-time exercise frequency

\begin{tabular}{|c|c|c|c|c|c|c|c|c|c|}
\hline \multirow[t]{3}{*}{ Variable } & \multicolumn{6}{|c|}{ Tertiles from low to high } & \multirow[t]{3}{*}{$c^{2}$} & \multicolumn{2}{|c|}{$F$ Fprobability $P$-value } \\
\hline & \multicolumn{2}{|l|}{ Low } & \multicolumn{2}{|l|}{ Medium } & \multicolumn{2}{|l|}{ High } & & & \\
\hline & Male & Female & Male & Female & Male & Female & & & \\
\hline $\begin{array}{l}\text { pFIT } \\
\text { Age } \\
\text { BMI } \\
\text { Smoking habit (all) }\end{array}$ & $\begin{array}{l}n=93 \\
46.1 \pm 15.2 \\
30.7 \pm 5.9 \\
11.7 \%\end{array}$ & $\begin{array}{l}n=246 \\
44.6 \pm 14.0 \\
29.3 \pm 6.7\end{array}$ & $\begin{array}{l}n=134 \\
43.7 \pm 16.1 \\
27.7 \pm 4.8^{*} \\
5.9 \%\end{array}$ & $\begin{array}{c}n=176 \\
46.6 \pm 15.9 \\
25.3 \pm 4.4^{*}\end{array}$ & $\begin{array}{l}n=166 \\
45.2 \pm 19.0 \\
25.5 \pm 3.4^{*} \\
4.0 \%\end{array}$ & $\begin{array}{c}n=183 \\
49.4 \pm 17.1^{*} \\
23.35 \pm 4.0^{*}\end{array}$ & $<0.001$ & $\begin{array}{r}0.550 \\
<0.001\end{array}$ & $\begin{array}{r}0.007 \\
<0.001\end{array}$ \\
\hline $\begin{array}{l}\text { ExFreq } \\
\text { Age } \\
\text { BMl } \\
\text { Smoking habit (all) }\end{array}$ & $\begin{array}{l}n=65 \\
47.8 \pm 15.2 \\
29.3 \pm 5.2 \\
12.1 \%\end{array}$ & $\begin{array}{l}n=149 \\
45.7 \pm 14.4 \\
28.8 \pm 6.9\end{array}$ & $\begin{array}{l}n=235 \\
45.1 \pm 17.4 \\
27.3 \pm 4.9 \\
7.6 \%\end{array}$ & $\begin{array}{c}n=337 \\
46.1 \pm 15.9 \\
26.2 \pm 5.5^{\star}\end{array}$ & $\begin{array}{c}n=93 \\
42.4 \pm 17.6 \\
26.5 \pm 4.8^{*} \\
1.4 \%\end{array}$ & $\begin{array}{l}n=119 \\
49.3 \pm 16.2 \\
23.9 \pm 4.4^{*}\end{array}$ & 0.008 & $\begin{array}{l}0.143 \\
0.002\end{array}$ & $\begin{aligned} & 0.120 \\
< & 0.001\end{aligned}$ \\
\hline
\end{tabular}

Perceived physical fitness level (pFIT), low (1-5 on a Likert scale), medium (6-7), and high (8-10). Leisure-time exercise frequency (ExFreq, days/week), low (<1 day/week), medium (1-4), high (2::5). Data are reported as mean \pm standard deviation.

${ }^{*} P<0.05$ compared with the low tertile.

BMI, body massindex.

Table2. Tertiles of perceived physical fitness from low to high

\begin{tabular}{|c|c|c|c|c|c|}
\hline \multirow[t]{2}{*}{ Variable } & \multicolumn{3}{|c|}{ Perceived physical fitness } & \multirow[t]{2}{*}{$P$ for trend } & \multirow[t]{2}{*}{ Factors selected } \\
\hline & Low & Medium & High & & \\
\hline $\begin{array}{l}\text { TNF- } a(\mathrm{pg} / \mathrm{mL}) \\
\text { MCP1 }(\mathrm{pg} / \mathrm{mL}) \\
\text { GCSF }(\mathrm{pg} / \mathrm{mL}) \\
\text { IL-10 }(\mathrm{pg} / \mathrm{mL}) \\
\text { WBC }\left(10^{9} / \mathrm{L}\right) \\
\text { FRAP }(\mathrm{mmol} / \mathrm{L})\end{array}$ & $\begin{array}{c}1.95(1.77-2.15) \\
169(163-175) \\
33.7(31.9-35.5) \\
1.65(1.47-1.86) \\
6.61(6.43-6.79) \\
592(577-606)\end{array}$ & $\begin{array}{c}1.91(1.72-2.12) \\
169(163-176) \\
32.2(30.4-34.1) \\
1.49(1.31-1.69) \\
6.35(6.15-6.55)^{*} \\
581(566-596)\end{array}$ & $\begin{array}{l}1.66(1.50-1.83)^{* \dagger} \\
171(165-177) \\
30.1(28.4-31.9)^{* \dagger} \\
1.43(1.24-1.64) \\
6.10(5.90-6.31)^{* \dagger} \\
585(571-600)\end{array}$ & $\begin{array}{c}0.003 \\
0.693 \\
<0.001 \\
0.076 \\
<0.001 \\
0.619\end{array}$ & $\begin{array}{l}\text { Age, pFIT, sex, smoking } \\
\text { Age, sex, smoking, pFIT } \\
\text { Sex, BMI, pFIT, smoking } \\
\text { Sex, BMI, pFIT, smoking } \\
\text { BMI, amoking, pFIT, age } \\
\text { Sex, age, BMI, pFIT }\end{array}$ \\
\hline
\end{tabular}

Perceived physical fitness (pFIT) level, low (1-5 on a Likert scale), medium (6-7), and high (8-10). Data for all but WBC and FRAP are presented as antilogs of least squares means after model adjustment $( \pm 95 \%$ confidence intervals). $P$-values for trend were adjusted for multiple tests by using false discovery rate.

*Significantly different from low fitness; ${ }^{\dagger}$ significantly different from medium fitness. Factors selected by the model are reported in rank order of importance.

TNF- $a$, tumor necrosis factor- $a$; MCP1, monocyte chemotactic protein-1; GCSF, granulocyte colony-stimulating factor; IL-10, interleukin-10; WBC, white blood cell count; FRAP, ferric reducing ability of plasma (expressed as ascorbic acid equivalents).

\section{Statistics}

Subject characteristics were contrasted between tertiles within gender using one-way analysis of variance for age and BMI, and the categorical variables, gender and smoking, were analyzed using chi-square analysis (Table 1). The general linear model (GLM) was used to examine the effect of perceived fitness (or ExFreq) on each plasma biomarker. The GLMSELECT procedure in SAS (version 9.1.3; SAS Institute, Inc., Cary, North Carolina, USA) was used to identify confounding variables. The candidate confounders that the GLMSELECT procedure selected from were age, gender, BMI, and smoking habit. Disease was added as a potential confounder to the model of each biomarker; however, the analyses suggest that disease does not affect the relationship between each biomarker and pFIT or ExFreq. For each marker, the model with the smallest Akaike's information corrected criterion was selected, and then a trend test was performed to study the effect of pFIT (or ExFreq) on the biomarker marker after adjusting for confounders. Benjamini-Hochberg method for false discovery rate correction in the MULTTEST procedure in SAS was used for multiple testing correction. Pairwise comparison was performed among the three pFIT (or ExFreq) levels. The normality of the residuals from each model was examined. When the normality assumption was violated, outliers with studentized residue $>2.5$ or $<-2.5$ were excluded, and when needed, natural $\log$ transformation on the response variable was performed. In Tables 2 and 3, $P$ for trend values represent adjusted pFIT and ExFreq data for the outcome measures, and in Figs 1, 2, and 3, P-values are listed separately for factors selected by the model.

\section{Results}

Table 1 summarizes age, BMI, and smoking habit data for males and females across tertiles from low to high pFIT levels and leisure-time ExFreq. BMI and smoking habit differed significantly across pFIT and ExFreq tertiles for both genders. Ages were similar across pFIT and ExFreq tertiles except for pFIT in females.

Across pFIT tertiles, CRP (Fig. 1a), IL-6 (Fig. 2a), TNF- $a$, GCSF, and WBC, but not MCP1 or IL-10 (Table 2) inflammation biomarkers, differed significantly after adjustment for factors selected by the model. 
Table3. Tertiles of leisure-time exercise frequency from low to high

\begin{tabular}{|c|c|c|c|c|c|}
\hline \multirow[t]{2}{*}{ Variable } & \multicolumn{3}{|c|}{ Leisure-time exercise frequency } & \multirow[t]{2}{*}{ Pfor trend } & \multirow[t]{2}{*}{ Factors selected } \\
\hline & Low & Medium & High & & \\
\hline $\begin{array}{l}\text { TNF- } a(\mathrm{pg} / \mathrm{mL}) \\
\text { MCP1 }(\mathrm{pg} / \mathrm{mL}) \\
\text { GCSF }(\mathrm{pg} / \mathrm{mL}) \\
\text { IL-10 }(\mathrm{pg} / \mathrm{mL}) \\
\text { WBC }\left(10^{9} / \mathrm{L}\right) \\
\text { FRAP }(\mathrm{mmol} / \mathrm{L})\end{array}$ & $\begin{array}{c}2.01(1.80-2.24) \\
169(163-176) \\
33.9(31.9-36.1) \\
1.62(1.44-1.82) \\
6.58(6.37-6.80) \\
577(560-596)\end{array}$ & $\begin{array}{c}1.80(1.64-1.96)^{*} \\
169(164-174) \\
32.1(30.6-34.7) \\
1.51(1.40-1.62) \\
6.38(6.21-6.55)^{\star} \\
584(573-595)\end{array}$ & $\begin{array}{l}1.86(1.65-2.10) \\
176(168-183)^{\dagger} \\
29.1(27.2-31.1)^{\star \dagger} \\
1.60(1.41-1.80) \\
6.12(5.89-6.35)^{\star \dagger} \\
604(587-623)^{\star \dagger}\end{array}$ & $\begin{array}{c}0.305 \\
0.133 \\
<0.001 \\
0.850 \\
<0.001 \\
0.047\end{array}$ & $\begin{array}{l}\text { Age, smoking, sex, BMl, ExFreq } \\
\text { Age, sex, smoking, ExFreq } \\
\text { Sex, BMI, ExFreq, smoking } \\
\text { BMl, sex, ExFreq } \\
\text { BMl, smoking, ExFreq, age } \\
\text { Sex, age, BMl, ExFreq }\end{array}$ \\
\hline
\end{tabular}

Leisure-time exercise frequency (ExFreq, days/week), low (<1 day/week), medium (1-4), and high (2::5). Data for all but WBC and FRAP are presented as antilogs of least squares means after model adjustment ( $\pm 95 \%$ confidence intervals). $P$-values for trend were adjusted for multiple tests using false discovery rate.

*Significantly different from low ExFreq; ${ }^{\text {}}$ significantly different from medium ExFreq. Factors selected by the model are reported in rank order of importance.

TNF- $a$, tumor necrosis factor- $a$; MCP1, monocyte chemotactic protein-1; GCSF, granulocyte colony-stimulating factor; IL-10, interleukin-10; WBC, white blood cell count; FRAP, ferric reducing ability of plasma (expressed as ascorbic acid equivalents).
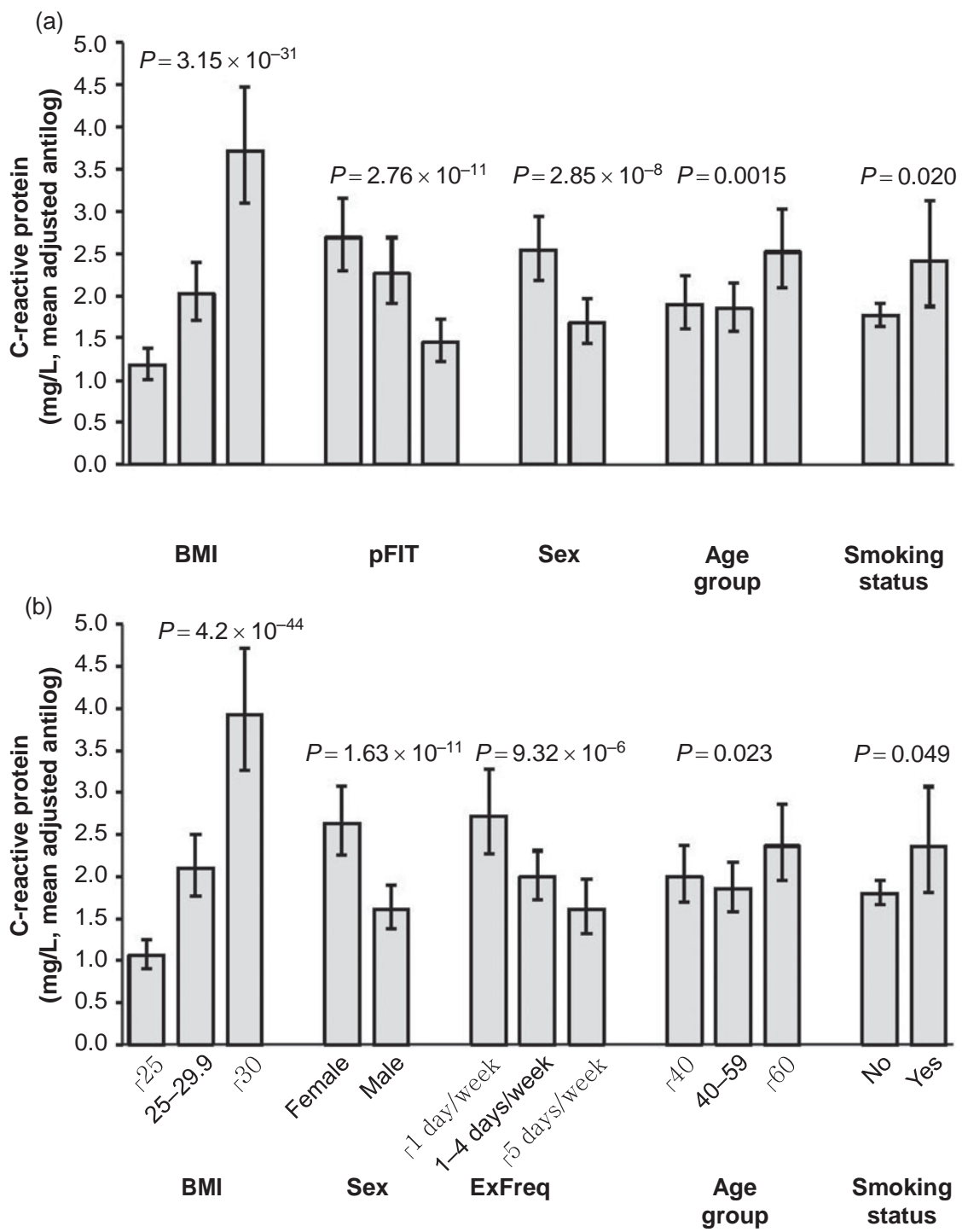

Fig. 1. Plasma C-reactive protein (CRP) for each of the factors selected into (a) the perceived physical fitness level (pFIT) and (b) the leisure-time exercise frequency (ExFreq) model. Data are antilogs of least squares means after model adjustment ( $\pm 95 \%$ confidence intervals). Factors selected are plotted in rank order of importance with the $P$ for trend value reported for each factor. 

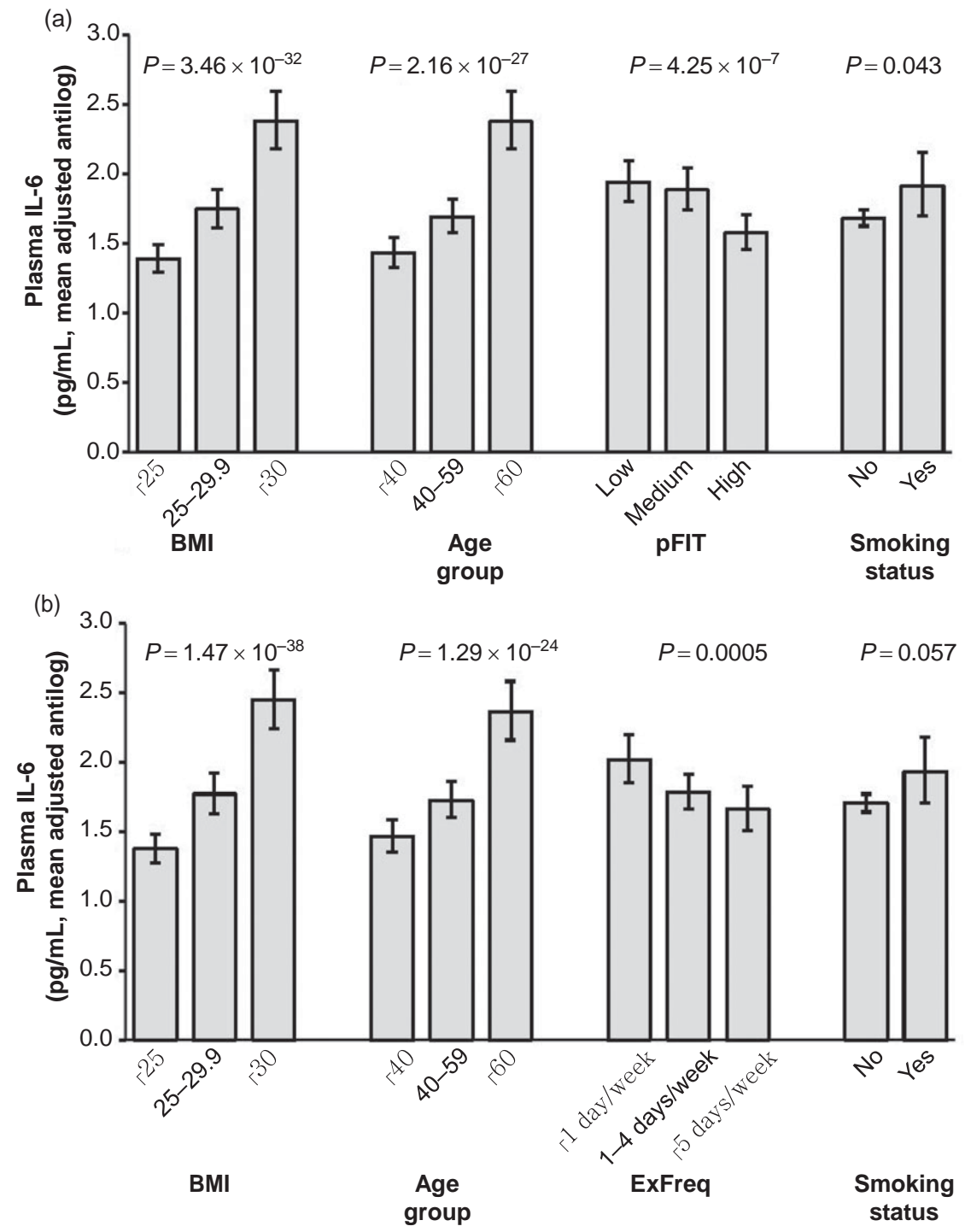

Fig. 2. Plasma interleukin (IL)-6 for each of the factors selected into (a) the perceived physical fitness level (pFIT) and (b) the leisure-time exercise frequency (ExFreq) model. Data are antilogs of least squares means after model adjustment ( $\pm 95 \%$ confidence intervals). Factors selected are plotted in rank order of importance with the $P$ for trend value reported for each factor.

Across ExFreq tertiles, CRP (Fig. 1b), IL-6 (Fig. 2b), GCSF, and WBC, but not TNF- $a$, MCP1, or IL-10 (Table 3) inflammation biomarkers, differed significantly after adjustment for factors selected by the model.

Across pFIT tertiles, $F_{2}$-isoprostanes (Fig. 3a) but not FRAP (Table 2), oxidative stress and capacity biomarkers, respectively, differed significantly after adjustment for factors selected by the model. Across ExFreq tertiles, $\mathrm{F}_{2}$-isoprostanes (Fig. $3 \mathrm{~b}$ ) and FRAP (Table 3) differed significantly after adjustment for factors selected by the model. None of the factors in the model (pFIT, ExFreq, age, sex, or smoking) were found to have a significant relationship with ORAC (see online Supporting Information).

For both pFIT and ExFreq tertiles, BMI was the factor selected first by the model for CRP, IL-6, WBC, and $\mathrm{F}_{2}$-isoprostanes, with sex selected first for GCSF and
FRAP, and age selected first for TNF- $a$ and MCP1 (Tables 2 and 3, Figs 1-3, see online Supporting Information). In general, the effect of the factors pFIT or ExFreq ranked second or third when compared with the other factors in the model.

\section{Discussion}

This community-based epidemiologic study was unique in that the relationship of both pFIT levels and leisuretime ExFreq on 10 measures of inflammation and oxidative stress was investigated in approximately 1000 male and female adults between the ages of 18 and 85 years. The primary finding of this study was that CRP, WBCs, IL-6, TNF- $a$, GCSF, and $\mathrm{F}_{2}$-isoprostanes were significantly lower when comparing high-to-low tertiles for both pFIT and ExFreq, after adjustment for multiple 

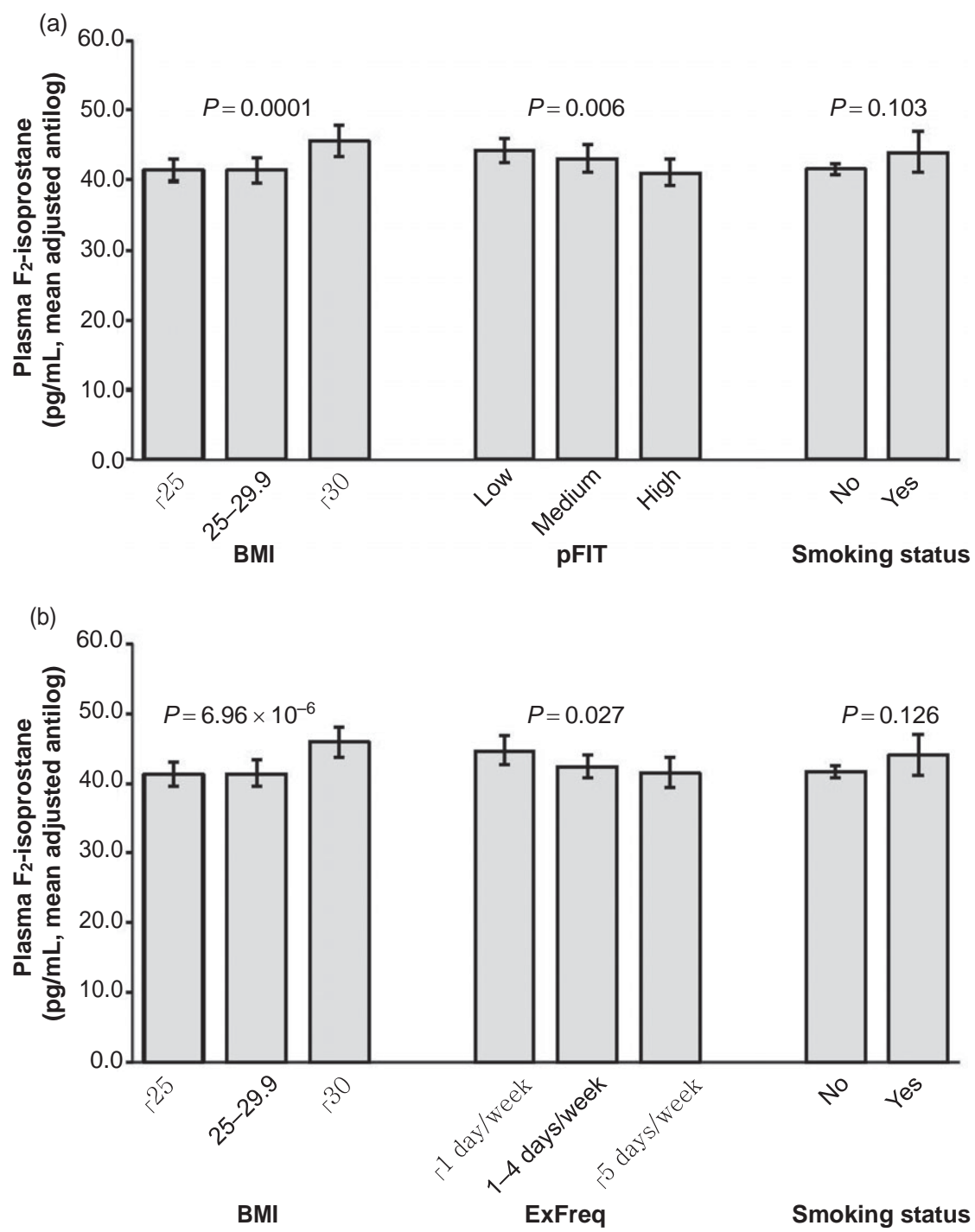

Fig. 3. Plasma $F_{2}$-isoprostane for each of the factors selected into (a) the perceived physical fitness level (pFIT) and (b) the leisure-time exercise frequency (ExFreq) model. Data are antilogs of least squares means after model adjustment ( $\pm 95 \%$ confidence intervals). Factors selected are plotted in rank order of importance with the $P$ for trend value reported for each factor.

confounders. These data indicate that in a large, heterogeneous sample of community-dwelling adults, an inverse relationship exists between multiple inflammation and oxidative stress biomarkers and pFIT and ExFreq even after adjustment for potential confounders. Overall, the effect of pFIT and ExFreq on CRP was strong, but for all other biomarkers, the effect was modest and generally ranked behind BMI or demographic factors in rank order of importance.

CRP is a well-characterized biomarker of chronic inflammation. CRP was significantly influenced by BMI, gender, age, and smoking status, but after adjustment for these factors, subjects in the upper tertiles for pFIT and ExFreq had 40-46\% lower CRP levels than those in the lower tertiles. Our data are in agreement with three other studies that have examined the influence of pFIT (Ford, 2002; Pitsavos et al., 2005) and ExFreq (King et al.,
2003) on CRP levels in heterogeneous populations. An elevated WBC is indicative of inflammation and is associated with adverse cardiovascular events (Danesh et al., 1998). WBCs were 7.0-7.7\% lower in subjects in the upper vs lower tertiles for pFIT and ExFreq in agreement with comparable studies (Ford, 2002; King et al., 2003; Pitsavos et al., 2005).

Our study also determined if pFIT and ExFreq were related to five plasma cytokines (IL-6, TNF- $a$, GCSF, MCP1, and IL-10). IL-6 was significantly lower in the high vs low pFIT (19\%) and ExFreq (18\%) tertiles. Likewise, TNF- $a$ was significantly lower in the high vs low pFIT (-15\%) and ExFreq (-8\%) tertiles. The magnitude of the inverse relationship between pFIT and IL- 6 or TNF- $a$ has been reported in studies with similar subject characteristics (Pitsavos et al., 2005; Arsenault et al., 2009), but this is the first such report for ExFreq. 
The cytokine GCSF is primarily secreted by monocytes and macrophages; however, other immune and nonimmune cell types can also secrete GCSF (Barreda et al., 2004). Neutrophil proliferation, differentiation, survival, and activation are regulated by GCSF (Barreda et al., 2004). Additionally, GCSF decreases pro-inflammatory cytokine secretion by activated monocytes and macrophages (Barreda et al., 2004). Plasma levels of GCSF increase in response to intense long- and short-duration exercise (Yamada et al., 2002; Pitsavos et al., 2005; Nieman et al., 2007). However, to our knowledge, this is the first study to determine if there is a link between pFIT or ExFreq and circulating GCSF levels. We found an inverse relationship between plasma GCSF levels and pFIT or ExFreq; plasma GCSF levels were significantly lower in the high pFIT $(-10.5 \%)$ and ExFreq (-14.2\%) tertiles. GCSF may support the anti-inflammatory actions of IL- 6 by inducing its release and inhibiting the release of IL- $1 b$ and TNF- $a$ (Suzuki et al., 2002; Nieman et al., 2005). In the community setting, the lower levels of GCSF reflect the lower degree of inflammation in the high pFIT and ExFreq tertiles.

MCP1 is a pro-inflammatory cytokine produced by macrophages and adipocytes and other cell types (Gustafson, 2010). MCP1 facilitates the infiltration and activation of monocytes, macrophages, and lymphocytes, and induces IL- 6 and TNF- $a$ secretion from adipocytes and macrophages, respectively (Gustafson, 2010). Like many other inflammatory cytokines, plasma MCP1 increases acutely in response to vigorous exercise (Nieman et al., 2005, 2007). Recent evidence suggests that short-term weight loss, but not exercise alone, can decrease plasma MCP1 levels in obese subjects (Christiansen et al., 2010). Our data indicate that plasma MCP1 is related to age, gender, and smoking but not pFIT, ExFreq, or BMI (see online Supporting Information).

IL-10, an exercise responsive (Nieman et al., 2005, 2007; Kadoglou et al., 2007) anti-inflammatory cytokine, inhibits synthesis of IL-12, thus inhibiting interferon- $g$ production. We found that plasma IL-10 is more related to gender and BMI than to pFIT of ExFreq (see online Supporting Information). Our data from a heterogeneous age-group extend the previous report of a lack of an apparent relationship between IL-10 and exercise habit in a cohort of elderly subjects (Elosua et al., 2005).

Obesity, old age, and chronic disease are associated with oxidative stress (Morrow, 2005; Voss \& Siems, 2006; Basu, 2008). Regular exercise can decrease lipid peroxidation, a measure of oxidative stress, in patients with cardiovascular and metabolic diseases (FisherWellman et al., 2009). We assessed oxidative stress with $\mathrm{F}_{2}$-isoprostanes, a sensitive and stable marker of lipid peroxidation (Morrow, 2005). Our data indicate that oxidative stress is significantly lower in high pFIT $(-7.1 \%)$ and high ExFreq $(-7.1 \%)$ tertiles. Plasma $\mathrm{F}_{2}$-isoprostanes have been correlated with BMI (Urakawa et al., 2003), but in our study, the effect of pFIT and ExFreq remained significant after adjusting for BMI. Exercise intervention studies have demonstrated that the training period must be of sufficient duration (e.g. greater than 8 weeks), and it must induce a substantial improvement in fitness to significantly decrease $\mathrm{F}_{2}$-isoprostanes (Schmitz et al., 2008; Campbell et al., 2010). To our knowledge, this is the first report of lower plasma $\mathrm{F}_{2}$-isoprostanes in high pFIT and ExFreq in a community setting.

Plasma is endowed with antioxidant enzymes, macromolecules, and small molecules, the sum of which is referred to as antioxidant capacity. Plasma antioxidant capacity can be modulated by diet, lifestyle, and disease state. FRAP did not differ across pFIT tertiles, but a trend for elevated levels $(4.7 \%)$ was found in the high ExFreq tertile. Overall, FRAP levels differed by gender, age, and BMI, with elevated levels found in older males with higher BMI (see online Supporting Information). Plasma ORAC, however, did not differ across pFIT and ExFreq tertiles and was not related to any of the measured confounders.

The primary strength of this study was the measurement of a broad array of inflammatory and oxidative stress biomarkers in a heterogeneous group of community-based adults, with GLM analysis of relationships to pFIT and ExFreq while controlling for important confounders. Indeed, six of the 10 measured biomarkers were significantly different across pFIT and/or ExFreq tertiles after adjusting for confounders. Our findings extend those reported in other populationbased, cross-sectional studies, but contrast with some randomized, exercise training studies that indicate no independent influence on inflammation and oxidative stress. One explanation is that the change in aerobic fitness is often small, the duration of training seldom extends beyond 6 months, and the number of subjects is relatively small in exercise intervention trials (Arsenault et al., 2009; Campbell et al., 2010; Christiansen et al., 2010; Church et al., 2010; Stewart et al., 2010; Thompson et al., 2010). In contrast, in large community, observational studies, the difference in ExFreq, and physical fitness levels far exceeds changes that are attainable in the training studies. For example, in our subjects, ExFreq varied widely (from $<1$ to $2:: 5$ days per week) between low and high tertiles, and more than half of our subjects who reported regular physical activity had maintained this lifestyle five or more years (data not shown).

\section{Perspectives}

Physical inactivity increases the incidence of numerous chronic diseases (Booth et al., 2000), many of which are associated with chronic low-grade inflammation and 
oxidative stress (Morrow, 2005; Basu, 2008; Beavers et al., 2010). Conversely, lean and highly fit endurance athletes have low levels of these biomarkers in the rested state. In this epidemiologic study of communitydwelling adults, pFIT levels and/or leisure-time ExFreq were associated with decreased levels of six of 10 of these factors even after adjustment for the important confounders BMI, age, gender, and smoking status. Previous studies have shown these relationships for many of the biomarkers studied, but this is the first study to establish a similar linkage to GCSF. In conclusion, our data indicate that the combination of low BMI and high levels of pFIT and/or self-reported aerobic activity frequency have a strong relationship with low levels of inflammation and oxidative stress.

Key words: cytokines, granulocyte colony-stimulating factor, $F_{2}$-isoprostanes, antioxidant capacity, leukocytes, body mass index, perceived physical fitness.

\section{Acknowledgements}

Sources of funding were Coca-Cola (Atlanta, Georgia, USA) and Quercegen Pharmaceuticals (Sudbury, Massachusetts, USA).

\section{Supporting information}

Additional Supporting Information may be found in the online version of this article:

Appendix S1. Model variables. Appendix S2. (Continued) pFIT GLM results. Appendix S3. (Continued) ExFreq GLM results.

Please note: Wiley-Blackwell are not responsible for the content or functionality of any supporting materials supplied by the authors. Any queries (other than missing material) should be directed to the corresponding author for the article.

\section{References}

Abramson JL, Vaccarino V. Relationship between physical activity and inflammation among apparently healthy middle-aged and older US adults. Arch Intern Med 2002: 162: 1286-1292.

Arsenault BJ, Cote M, Cartier A, Lemieux I, Despres JP, Ross R, Earnest CP, Blair SN, Church TS. Effect of exercise training on cardiometabolic risk markers among sedentary, but metabolically healthy overweight or obese post-menopausal women with elevated blood pressure. Atherosclerosis 2009: 207: 530-533.

Barreda DR, Hanington PC, Belosevic M. Regulation of myeloid development and function by colony stimulating factors. Dev Comp Immunol 2004: 28: 509-554.

Basu S. F2-isoprostanes in human health and diseases: from molecular mechanisms to clinical implications. Antioxid Redox Signal 2008: 10: 14051434.

Beavers KM, Brinkley TE, Nicklas BJ. Effect of exercise training on chronic inflammation. Clin Chim Acta 2010: 411: 785-793.

Booth FW, Gordon SE, Carlson CJ, Hamilton MT. Waging war on modern chronic diseases: primary prevention through exercise biology. J Appl Physiol 2000: 88: 774-787.

Campbell PT, Gross MD, Potter JD, Schmitz KH, Duggan C, Anne A, Ulrich CM. Effect of exercise on oxidative stress: a 12-month randomized, controlled trial. Med Sci Sports Exerc 2010: 42: 1448-1453.

Christiansen T, Paulsen SK, Bruun JM, Pedersen SB, Richelsen B. Exercise training versus diet-induced weight-loss on metabolic risk factors and inflammatory markers in obese subjects: a 12-week randomized intervention study. Am J Physiol Endocrinol Metab 2010: 298: E824E831.

Church TS, Earnest CP, Thompson AM, Priest E, Rodarte RQ, Sanders T, Ross R, Blair SN. Exercise without weight loss does not reduce C-reactive protein: the INFLAME Study. Med Sci Sports Exerc 2010: 42: 708-716.

Colbert LH, Visser M, Simonsick EM, Tracy RP, Newman AB, Kritchevsky SB, Pahor M, Taaffe DR, Brach J, Rubin S, Harris TB. Physical activity, exercise, and inflammatory markers in older adults: findings from the Health, Aging and Body Composition Study. J Am Geriatr Soc 2004: 52: 1098-1104.

Danesh J, Collins R, Appleby P, Peto R. Association of fibrinogen, C-reactive protein, albumin, or leukocyte count with coronary heart disease: meta-analyses of Prospective Studies. JAMA 1998: 279: 1477-1482.

Elosua R, Bartali B, Ordovas JM, Corsi AM, Lauretani F, Ferrucci L. Association between physical activity, physical performance, and inflammatory biomarkers in an elderly population: the InCHIANTI study. J Gerontol A Biol Sci Med Sci 2005: 60: 760-767.

Esmaillzadeh A, Kimiagar M, Mehrabi Y, Azadbakht L, Hu FB, Willett WC. Fruit and vegetable intakes, $C$-reactive protein, and the metabolic syndrome. Am J Clin Nutr 2006: 84: 1489-1497.
Fisher-Wellman K, Bell HK, Bloomer RJ. Oxidative stress and antioxidant defense mechanisms linked to exercise during cardiopulmonary and metabolic disorders. Oxid Med Cell Longev 2009: 2: 43-51.

Ford ES. Does exercise reduce inflammation? Physical activity and C-reactive protein among U.S. adults. Epidemiology 2002: 13: 561-568.

Gerber M, Brand S, Holsboer-Trachsler E, Puhse U. Fitness and exercise as correlates of sleep complaints: is it all in our minds? Med Sci Sports Exerc 2010: 42: 893-901.

Gustafson B. Adipose tissue, inflammation and atherosclerosis. J Atheroscler Thromb 2010: 17: 332-341.

Heinz SA, Henson DA, Nieman DC, Austin MD, Jin F. A 12-week supplementation with quercetin does not affect natural killer cell activity, granulocyte oxidative burst activity or granulocyte phagocytosis in female human subjects. Br J Nutr 2010: 104: 849-857.

Holt EM, Steffen LM, Moran A, Basu S, Steinberger J, Ross JA, Hong CP, Sinaiko AR. Fruit and vegetable consumption and its relation to markers of inflammation and oxidative stress in adolescents. J Am Diet Assoc 2009: 109: 414-421.

Kadoglou NP, Iliadis F, Angelopoulou N, Perrea D, Ampatzidis G, Liapis CD, Alevizos M. The anti-inflammatory effects of exercise training in patients with type 2 diabetes mellitus. Eur J Cardiovasc Prev Rehabil 2007: 14: 837-843. 
Kelley GA, Kelley KS. Effects of aerobic exercise on C-reactive protein, body composition, and maximum oxygen consumption in adults: a meta-analysis of randomized controlled trials. Metabolism 2006: 55: 15001507.

King DE, Carek P, Mainous AG, 3rd, Pearson WS. Inflammatory markers and exercise: differences related to exercise type. Med Sci Sports Exerc 2003: 35: 575-581.

Kohut ML, McCann DA, Russell DW, Konopka DN, Cunnick JE, Franke WD, Castillo MC, Reighard AE, Vanderah E. Aerobic exercise, but not flexibility/resistance exercise, reduces serum IL-18, CRP, and IL-6 independent of beta-blockers, BMI, and psychosocial factors in older adults. Brain Behav Immun 2006: 20: 201209.

Milton K, Bull FC, Bauman A. Reliability and validity testing of a single-item physical activity measure. Br J Sports Med 2011: 45: 203-208.

Morrow JD. Quantification of isoprostanes as indices of oxidant stress and the risk of atherosclerosis in humans. Arterioscler Thromb Vasc Biol 2005: 25: 279-286.

National Center for Health Statistics (U.S.). National Health Interview Survey : research for the 1995-2004 redesign. Hyattsville, MD: U.S. Dept. of Health and Human Services, Centers for Disease Control and Prevention, National Center for Health Statistics, 1999.

Nieman DC, Dumke CL, Henson DA, McAnulty SR, Gross SJ, Lind RH. Muscle damage is linked to cytokine changes following a $160-\mathrm{km}$ race.
Brain Behav Immun 2005: 19: 398-403.

Nieman DC, Henson DA, Davis JM, Dumke CL, Gross SJ, Jenkins DP, Murphy EA, Carmichael MD, Quindry JC, McAnulty SR, McAnulty LS, Utter AC, Mayer EP. Quercetin ingestion does not alter cytokine changes in athletes competing in the Western States Endurance Run. J Interferon Cytokine Res 2007: 27: 1003-1011.

Pitsavos C, Panagiotakos DB, Chrysohoou C, Kavouras S, Stefanadis C. The associations between physical activity, inflammation, and coagulation markers, in people with metabolic syndrome: the ATTICA study. Eur J Cardiovasc Prev Rehabil 2005: 12: 151-158.

Plante TG, Lantis A, Checa G. The influence of perceived versus aerobic fitness on psychological health and physiological stress responsivity. Int J Stress Manage 1998: 5: 141-156.

Schmitz KH, Warren M, Rundle AG, Williams NI, Gross MD, Kurzer MS. Exercise effect on oxidative stress is independent of change in estrogen metabolism. Cancer Epidem Biomar 2008: 17: 220-223.

Selvin E, Paynter NP, Erlinger TP. The effect of weight loss on C-reactive protein: a systematic review. Arch Intern Med 2007: 167: 31-39.

Shanely RA, Knab AM, Nieman DC, Jin F, McAnulty SR, Landram MJ. Quercetin supplementation does not alter antioxidant status in humans. Free Radical Res 2010: 44: 224-231.

Stewart LK, Earnest CP, Blair SN, Church TS. Effects of different doses of physical activity on c-reactive protein among women. Med Sci Sports Exerc 2010: 42: 701-707.
Stewart LK, Flynn MG, Campbell WW, Craig BA, Robinson JP, Timmerman KL, McFarlin BK, Coen PM, Talbert E. The influence of exercise training on inflammatory cytokines and C-reactive protein. Med Sci Sports Exerc 2007: 39: 1714-1719.

Suzuki K, Nakaji S, Yamada M, Totsuka M, Sato K, Sugawara K. Systemic inflammatory response to exhaustive exercise. Cytokine kinetics. Exerc Immunol Rev 2002: 8: 6-48.

Thompson D, Markovitch D, Betts JA, Mazzatti D, Turner J, Tyrrell RM. Time course of changes in inflammatory markers during a 6-mo exercise intervention in sedentary middle-aged men: a randomized-controlled trial. J Appl Physiol 2010: 108: 769-779.

Urakawa H, Katsuki A, Sumida Y, Gabazza EC, Murashima S, Morioka K, Maruyama N, Kitagawa N, Tanaka T, Hori Y, Nakatani K, Yano Y, Adachi Y. Oxidative stress is associated with adiposity and insulin resistance in men. J Clin Endocrinol Metab 2003: 88: 4673-4676.

Vincent HK, Taylor AG. Biomarkers and potential mechanisms of obesity-induced oxidant stress in humans. Int J Obes (Lond) 2006: 30: 400-418.

Voss P, Siems W. Clinical oxidation parameters of aging. Free Radical Res 2006: 40: 1339-1349.

Yamada M, Suzuki K, Kudo S, Totsuka M, Nakaji S, Sugawara K. Raised plasma G-CSF and IL-6 after exercise may play a role in neutrophil mobilization into the circulation. J Appl Physiol 2002: 92: 1789-1794. 